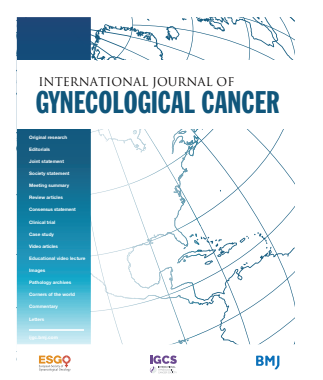

\title{
Infection versus cancer: management of actinomyces mimicking cervical cancer or ovarian cancer
}

Discussant:
Diego Odetto
Gynecology Oncology, Hospital Italiano de Buenos Aires, Buenos Aires, Argentina

Presenter:

Myriam Perrotta

Gynecology Oncology, Hospital Italiano de Buenos Aires, Buenos Aires, Argentina

Presenter:

Jose Martin Saadi

Gynecology Oncology, Hospital Italiano de Buenos Aires, Buenos Aires, Argentina

Radiologist:

Carolina Beatriz Chacon

Radiology, Hospital Italiano de Buenos Aires, Buenos Aires, Argentina

Radiologist:

Pamela Ines Causa Andrieu

Radiology, Hospital Italiano de Buenos Aires, Buenos Aires, Argentina

Radiology, Memorial Sloan Kettering Cancer Center, New York, New York, USA

Pathologist:

Alejandra Wernicke

Department of Pathology, Hospital Italiano de Buenos Aires, CABA, Buenos Aires, Argentina

Pathologist:

Marie Catherine Saez Perrotta

Department of Pathology, Hospital Italiano de Buenos Aires, CABA, Buenos Aires, Argentina

Correspondence to

Dr Diego Odetto, Gynecology Oncology, Hospital Italiano de Buenos Aires, Buenos Aires, Argentina; diego.odetto@ hospitalitaliano.org.ar

Accepted 6 July 2020 Published Online First 4 August 2020

Check for updates

(C) IGCS and ESGO 2020. № commercial re-use. See rights and permissions. Published by BMJ.

To cite: 0 detto $D$, Perrotta $M$, Saadi JM, et al. Int J Gynecol Cancer 2020;30:1638-1643.

\section{CASE PRESENTATIONS}

\section{Case 1}

A 34-year-old G2P2 patient presented to the emergency room with a 2-day history of lower abdominal pain, vaginal bleeding, and fever. The patient had no relevant past medical history and no previous surgeries. Menarche occurred at age 12 years, coitarche at age 19 years, with a history of five lifetime sexual partners. The patient had undergone a copper intrauterine device (IUD) placement after a vaginal delivery 2 years before this presentation. The patient reported no history of smoking or drug abuse. The patient's past gynecologic history was significant for an abnormal Pap smear with a highgrade squamous intraepithelial lesion, with a normal colposcopy examination, diagnosed 3 months before this episode. At that time, a cervical polyp was removed and no evidence of pre-invasive disease or cancer was found. Pathology revealed evidence of actinomyces (Figure $1 \mathrm{~A}, \mathrm{~B})$. The patient was recommended oral antibiotics (amoxicillin $875 \mathrm{mg}$-clavulanic acid $125 \mathrm{mg}$ for 3 weeks) but was not compliant with treatment and did not follow up. At that time, based on published data, we decided not to remove the IUD. ${ }^{1}$

Three months after the initial visit to the pre-invasive clinic, the patient was evaluated in the emergency room with the symptoms described above. On presentation, the patient had grade 2 dyspnea associated with tachycardia (90 beats per minute) and her temperature was $38^{\circ} \mathrm{C}\left(100.4^{\circ} \mathrm{F}\right)$. Physical examination showed evidence of lower abdominal, bilateral adnexal, and cervical tenderness. Speculum evaluation showed an enlarged friable and edematous cervix. The colposcopy examination revealed transformation zone type 3 with a non-visible squamocolumnar junction and signs of cervicitis. Laboratory studies showed a white blood cell count $18000 / \mathrm{mm}^{3}$, hemoglobin $7.1 \mathrm{~g} / \mathrm{dL}$, and platelet count $674000 / \mathrm{mm}^{3}$. A transvaginal ultrasound was ordered. 


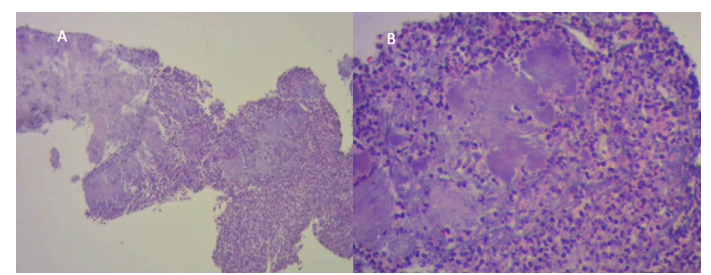

Figure 1 Endocervical biopsy showed wooly aggregates of filaments admixed with inflammatory debris, consistent with actinomyces. Hemotoxylin and eosin $(H \& E)$ staining $\times 10(A)$ and $\times 40(B)$.

\section{DR CHACON}

The transvaginal ultrasound (Figure 2) revealed a well-defined and isoechoic to hypoechoic lesion with increased vascularization on Doppler examination (arrow) involving both cervical lips, $3.7 \times 3.0$ $\times 2.5 \mathrm{~cm}$. To better characterize the cervical lesion delineated in the ultrasound, a pelvic magnetic resonance image (MRI) with and without gadolinium was ordered.

The pelvic MRI (Figure 3A-F), sagittal T2-weighted (A) shows a cervical mass with an intermediate signal on T2 and welldefined borders (arrow). Axial T2-weighted (B) shows that the mass infiltrates the deep myocervix, without involving the parametrium (arrow). Such mass is hypoenhancing (arrow) on sagittal $T 1$ post-contrast images (C). Also, it has homogenous restricted diffusion (arrows) on the diffusion-weighted image (DWI) (D) with its respective attenuation diffusion coefficient $(A D C)$ (E). Moreover, sagittal (A) and axial (F) T2-weighted images show associated surrounding inflammatory findings, such as a probably inflammatory nodule in the vesicouterine pouch, $1.5 \times 1.5 \mathrm{~cm}$, indicated with a star in $(A)$, and thickening of both fallopian tubes, indicated with arrows in (F).

The intermediate T2 signal intensity and the restricted diffusion are indicative of the cellularity of the cervical mass. Still, the conjunction of well-defined borders of the mass and the surrounding inflammatory findings possibly represented a pelvic inflammatory process involving the cervix. Therefore, the two possible diagnoses in such a scenario are cervical malignancy and cervicitis.

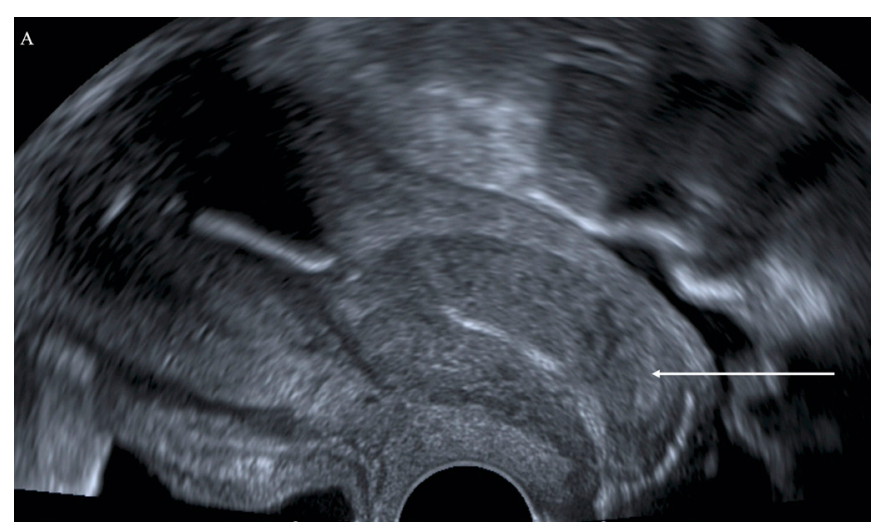

Figure 2 Transvaginal ultrasound showing the cervical mass.

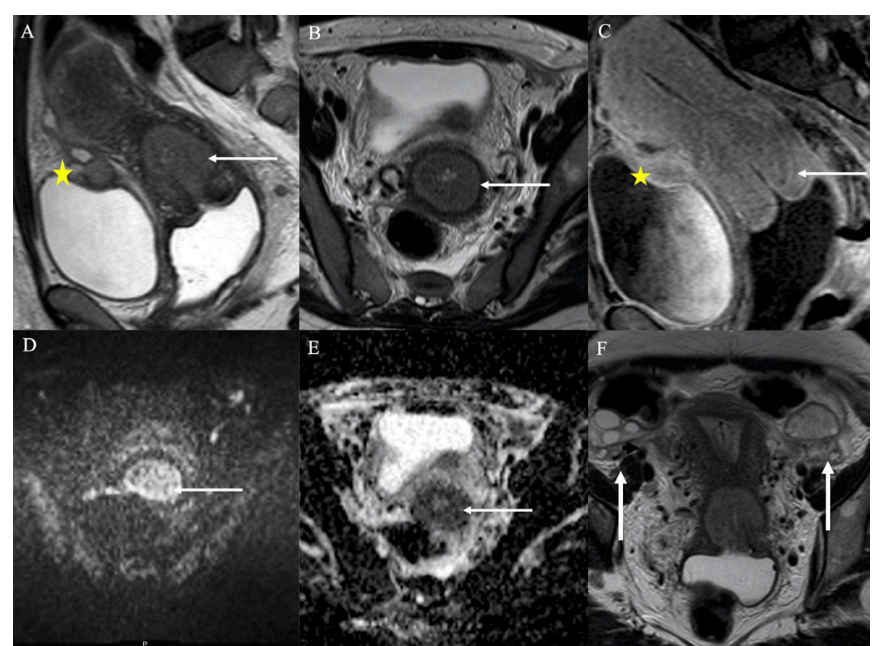

Figure 3 Pelvic magnetic resonance imaging (MRI) with and without gadolinium at diagnosis for initial characterization and staging of the cervical mass. (A) Sagittal T2-weighted. (B) Axial T2-weighted. (C) Axial T1-gadolinium. (D) Diffusionweighted images (DWI) B value $1000 \mathrm{~mm}^{3} / \mathrm{s}$. (E) Attenuation diffusion coefficient (ADC). (F) Axial T2- weighted.

\section{DR ODETTO}

Please provide details regarding the differential diagnosis at this time and the reasons to support such considerations? What recommendations were discussed with the patient at this time?

In a young woman with fever, lower abdominal pain, presumed collection in both tubes, and free fluid in the cul-de-sac, associated with a history of recently diagnosed actinomyces, the first consideration on the differential diagnosis was a pelvic inflammatory disease. However, the lesion in the cervix as demonstrated in the imaging studies raised concerns regarding a malignancy, and this was particularly so given the prior history of high-grade intraepithelial lesions. Based on the history of bacterial infection, the elevated white blood cell count, fever, and abdominal pain, we considered that the patient most likely had a diagnosis of pelvic inflammatory disease and recommendation for immediate laparoscopy was suggested. To our knowledge, there were no published data on anaerobic Gram-positive bacteria (actinomyces) in the cervix, mimicking a International Federation of Gynecology and Obstetrics (FIGO) stage I cervical cancer.

The patient was taken to the operating room with a plan for laparoscopic bilateral salpingectomy and lavage. In addition, an examination under anesthesia was planned to address the findings in the cervix. Examination under anesthesia revealed a 5-6 cm round firm cervix. Speculum evaluation showed an enlarged friable and edematous cervix. Cervical biopsies were performed at the four quadrants around the transformation zone, and the IUD was removed and sent for culture. The surgical evaluation revealed bilateral hydrosalpinx with $50 \mathrm{~mL}$ purulent fluid in the cul-de-sac. A bilateral salpingectomy was performed. The estimated blood loss was $200 \mathrm{~mL}$, and surgical time was $110 \mathrm{~min}$ with no intra-operative complications.

After surgery, the patient was started on intravenous antibiotics (ampicillin-sulbactam $1.5 \mathrm{~g}$ every 6 hours). The patient's condition gradually improved, and the fever subsided 2 days after the 


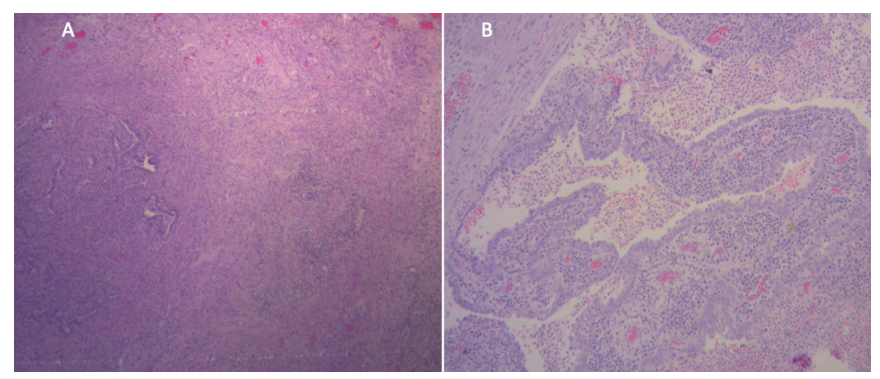

Figure 4 (A) Fallopian tube with acute salpingitis.

Hemotoxylin and eosin $(\mathrm{H} \& \mathrm{E})$ staining $\times 4$. The mucosa shows distorted architecture and dense inflammatory infiltrate that affects the myosalpinx. (B) Marked distortion of the plicae with dense mixed inflammatory infiltrate. H\&E staining $\times 4$.

initiation of therapy. The peritoneal fluid culture was as follows: bacteriological: Enterococcus faecalis; tuberculosis culture: negative; mycological: Candida albicans; antibiogram: sensitive to gentamicin-streptomycin. The IUD culture was as follows: bacteriological: Streptococcus agalactiae; mycological: Candida albicans; antibiogram: sensitive to amoxicillin-gentamicin.

\section{DR WERNICKE}

The bilateral salpingectomy specimen showed that both fallopian tubes were enlarged and edematous, measuring 9 and $8 \mathrm{~cm}$ in length, and the serosa appeared congestive and rough. The cut section revealed a dilated lumen of $0.5 \mathrm{~cm}$ diameter and erythematous mucosa. Histologically, there was a loss of normal mucosal architecture with a dense mixed inflammatory infiltrative area of ulceration and luminal fibrinous debris, consistent with acute salpingitis (Figure 4A,B).

The cervical biopsy showed abundant fibrinoid debris admixed with neutrophils, lymphocytes, and plasma cells, and isolated strips of squamous epithelium with a total loss of polarity, significant atypia in the basal and parabasal layers, as well as abnormal mitotic figures and superficial koilocytosis. Immunohistochemical stains for p16 showed strong and diffuse positivity. These microscopic findings were consistent with high-grade intraepithelial lesions.

The patient was discharged on post-operative day 7 and completed six additional weeks of amoxicillin $1 \mathrm{~g}$ every 8 hours plus doxycycline $100 \mathrm{mg}$ every 12 hours for 3 weeks. After the patient was discharged home, during her post-operative clinic visit, to determine a definite diagnosis of the endocervical lesion, a loop electrosurgical excision procedure (LEEP) conization was performed. Pelvic examination showed the same enlarged and firm cervix as previously seen but with no tenderness.

\section{DR WERNICKE}

Pathologic examination of the conization exhibited, in the transformation zone, a squamous epithelium with complete loss of polarity, atypia, and numerous mitosis consistent with a high-grade intraepithelial lesion. The underlying stroma showed edema, a mixed inflammatory infiltrate, and lymphoid follicles with the prominent germinal center. These histopathologic findings were consistent

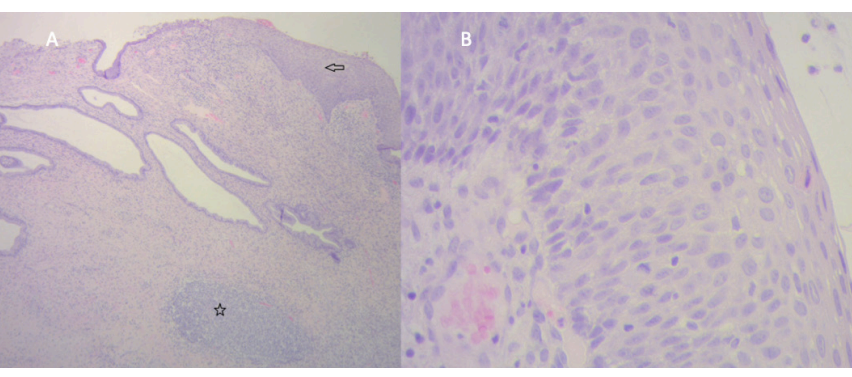

Figure 5 Loop electrosurgical excision procedure (LEEP) specimen. (A) The transformation zone shows squamous epithelium with immature basaloid cells extending to the middle of the epithelium, high mitotic figures, and superficial koilocytosis (arrow). The underlying stroma shows edema, a mixed inflammatory infiltrate, and lymphoid follicles with prominent germinal center (star). Hemotoxylin and eosin (H\&E) staining $\times 10$. (B) Detail of the dysplastic epithelium, with loss of polarity, high mitotic figures, and parakeratosis. H\&E staining $\times 40$.

with the diagnosis of the high-grade squamous intraepithelial lesion and chronic cervicitis (Figure 5A,B).

The patient was followed on a monthly basis. Three months after the antibiotics were completed, the cervix was macroscopically normal; the colposcopy examination showed a completely normal squamocolumnar junction with no other findings. A Pap smear and a human papillomavirus test were performed, and both were normal. A pelvic MRI was requested to evaluate the status of the cervical lesion and this was completely normal (Figure 6A-D).

\section{Case 2}

A 42-year-old G1P1 patient presented with a 45-day history of lower abdominal pain, abdominal distension, anorexia, and unintentional 15 pound weight loss.

Past medical and surgical history was unremarkable. Recent gynecologic history was significant for placement of a copper IUD at the age of 33 years. The patient had a prior exchange of IUD 4 years before this presentation. At the time of presentation, vital signs were within normal range. On pelvic examination, although no enlarged tumor was palpable, uterine and right adnexal tenderness was noticed. There was no evidence of cervical or vaginal discharge or bleeding.

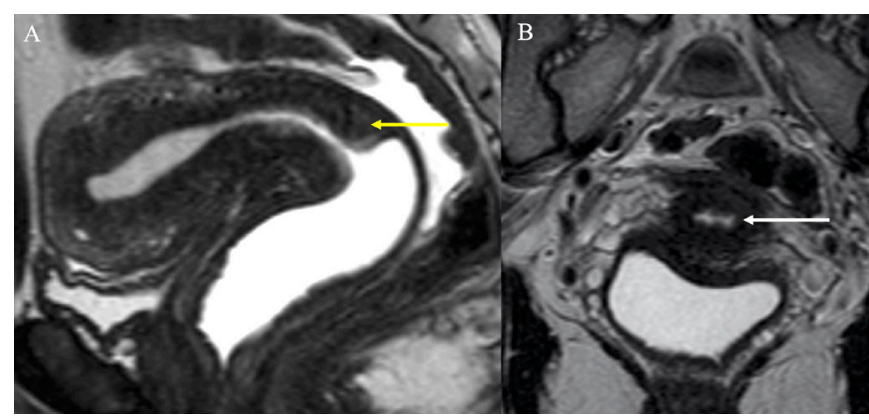

Figure 6 Magnetic resonance imaging (MRI) after antibiotic treatment. (A) Sagittal T2-weighted. (B) Axial T2-weighted. This images show the complete resolution of the cervical mass. The arrows in $(A)$ and $(B)$ indicate the normal cervix, without any lesion delineated. 


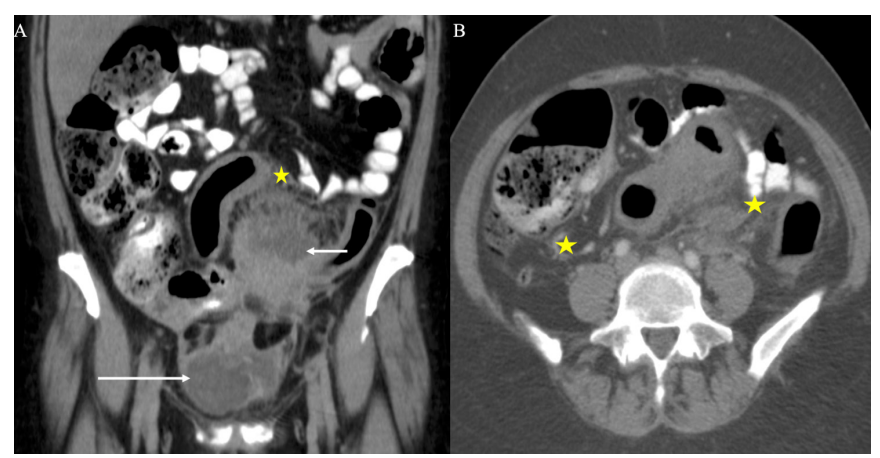

Figure 7 Computed tomography (CT) scan with oral and intravenous contrast $(A, B)$ showing an abdominopelvic collection (short arrows) and an adnexal mass (long arrows). The surrounding inflammatory changes are indicated with stars. (A) CT abdomen and pelvis, portal-venous phase, coronal plane. (B) CT abdomen and pelvis, portal-venous phase, axial plane.

Laboratory studies showed a white blood cell count of $17500 /$ $\mathrm{mm}^{3}$, hemoglobin $11.2 \mathrm{~g} / \mathrm{dL}$, platelet count $350000 / \mathrm{mm}^{3}$. Serum markers showed a carcinoembryonic antigen (CEA) level of $2 \mathrm{ng} /$ $\mathrm{mL}$, alpha-fetoprotein $3.4 \mathrm{ng} / \mathrm{mL}, \mathrm{CA} 125120 \mathrm{U} / \mathrm{mL}$, and CA19-9 $30 \mathrm{U} / \mathrm{mL}$. An initial chest radiograph showed no evidence of cardiopulmonary abnormalities. A computed tomography (CT) scan was requested with the findings described below, and subsequently a pelvic MRI with and without gadolinium was obtained.

\section{DR CAUSA ANDRIEU}

Initial CT of the abdomen and pelvis with intravenous contrast (Figure $7 \mathrm{~A}, \mathrm{~B}$ ) on the portal-venous phase, coronal plane $(A)$, shows two rim-enhancing and fluid-containing lesions. One was superior in the mid-abdomen close to bowel loops (short arrow), and the other was inferior in the right pelvis (long arrow). Moreover, portalvenous phase, axial plane (B) shows marked inflammatory findings such as trace-free fluid, fat stranding, enlarged lymph nodes, and mesenteric vascular engorgement; all of these are indicated with stars. These findings possibly represent abdominopelvic and adnexal collections.

We requested further MRI for better characterization. Pelvic MRI (Figure 8A-F) on axial T2-weighted (A) shows the above-mentioned inferior lesion in the right pelvis marked with long arrows, localized in the right adnexa. It is a tubular structure with a regularly thickened wall with an intense enhancement on post-contrast images (C), suggesting active inflammation. It is filled with a fluid that has a high signal on T2 (B) and with restricted diffusion (E,F), probably due to the high content in protein or pus. The right ovary was not delineated. Sagittal T2-weighted (B) shows the above-mentioned superior lesion marked with short arrows, localized in the lower abdomen and pelvis. It is a cystic, bilobed lesion filled with a heterogeneous fluid-fluid level. Such fluid has a high signal on T2-weighted $(A, B)$ and with restricted diffusion $(E, F)$, also suggestive of a high content of proteins or pus. The wall of the cystic lesion has intense enhancement on post-contrast images $(C, D)$, indicative of active inflammation.

Both lesions are surrounded by extensive inflammatory findings such as fat stranding and trace-free fluid, indicated with stars in

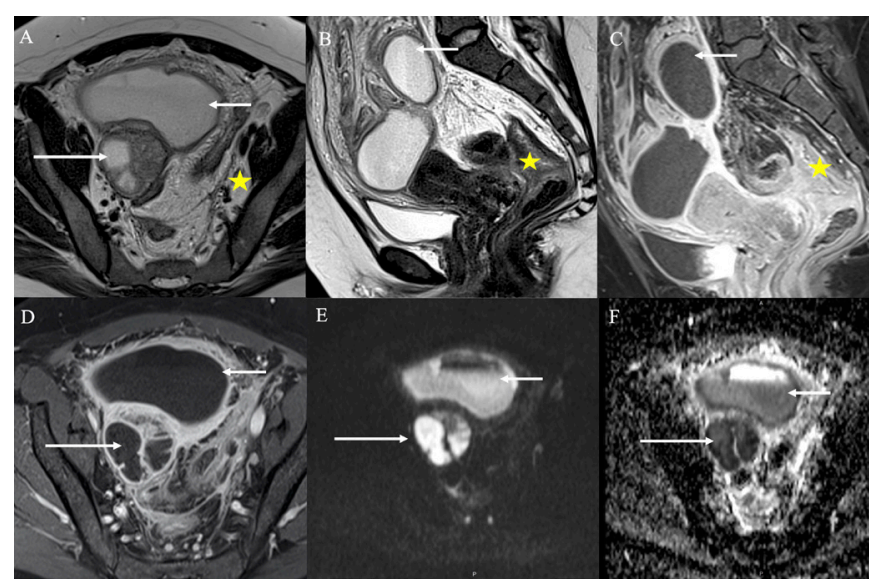

Figure 8 Pelvic magnetic resonance imaging (MRI) for further characterization, which shows a probably abdominopelvic abscess (short arrow) and a right tuboovarian abscess (long arrow). The surrounding inflammatory changes are indicated with stars. (A) Axial T2-weighted. (B) Sagittal T2-weighted. (C) Sagittal T1-gadolinium. (D) Axial T1- gadolinium. (E) Diffusion-weighted images (DWI) B value $1000 \mathrm{~mm}^{3} / \mathrm{s}$. (F) Attenuation diffusion coefficient (ADC)

(A-C). Also, the image shows an inflammatory nodule in the pouch of Douglas, infiltrating the anterior rectum wall, indicated with a star in (B). In conjunction, these findings probably represent an abdominopelvic abscess (short arrows) and a right tubo-ovarian abscess (long arrows).

\section{DR ODETTO}

Based on these imaging findings and serum tumor markers, what was the differential diagnosis for the patient at this time? What recommendations should be discussed with the patient?

Given the patient's age, unintentional 15 pound weight loss, chronic lower abdominal pain, with a suspicious mass on images, along with an elevated CA125, the consideration was for a newly diagnosed ovarian carcinoma. However, the fact that the patient did not have a personal or family history of cancer and that the images were not suggestive of typical findings of epithelial or germinal ovarian tumor, such as ascites, carcinomatosis, or omental caking, one should consider another process such as a chronic infection caused by tuberculosis, nocardiosis, or actinomyces. These are rare conditions whose symptoms may mimic malignant pelvic tumors, spread progressively and continuously, usually associated with weight loss, non-specific pelvic or abdominal pain, vaginal bleeding or discharge, and, in the case of actinomyces, associated with prolonged use of an IUD. ${ }^{2-4}$

With these two potential diagnoses, it was decided to take the patient to the operating room for an exploratory laparotomy. As a result of what was oberved on the MRI images, regardless of whether the origin was due to an infectious or tumor cause, the patient was informed about the high possibility of a bowel resection. Although the role of cytoreduction in ovarian cancer is well established, ${ }^{5}$ there is a gap in the knowledge as to when this is necessary, when considering a chronic infection by actinomyces. ${ }^{3}$ 


\section{Case study}

Intra-operative findings showed no evidence of carcinomatosis in the upper abdomen or the pelvis. There was evidence of severe adhesions with a sealed pelvis and a right $6 \mathrm{~cm}$ ovarian mass densely adherent to the ileocecal junction, small bowel, and the anterior wall of the rectum. Several biopsies were performed of the capsule of the ovary and cultures of the peritoneal fluid were obtained. Frozen section was performed and could not rule out ovarian cancer or peritoneal cancer due to an extensive inflammatory process. An en bloc resection of the terminal ileum, the ascending colon up to $7 \mathrm{~cm}$ below the hepatic angle was performed, along with bilateral salpingo-oophorectomy, hysterectomy, and omentectomy in order to remove the abscess altogether. Subsequently, an ileoascending-end to lateral anastomosis was performed. Estimated blood loss was $900 \mathrm{~mL}$, and one unit of packed red blood cells was administered. The operative time was $310 \mathrm{~min}$ and there were no intra-operative complications.

The patient was administered intravenous antibiotics with ampicillin-sulbactam $1.5 \mathrm{~g}$ every 6 hour for 8 days. The peritoneal fluid culture showed: bacteriological: coccus(+) Staphylococcus epidermidis; tuberculosis culture: negative; mycological: negative; antibiogram: sensitive to vancomycin-rifampicin. The patient was discharged on post-operative day 8 and completed seven additional weeks of amoxicillin $1 \mathrm{~g}$ every 8 hours plus doxycycline $100 \mathrm{mg}$ every 12 hours for 3 weeks until the pathologic diagnosis confirmed findings consistent with actinomycosis.

\section{DR SAEZ PERROTTA}

Macroscopic gross pathology examination of the formalin-fixed tissues included total hysterectomy with bilateral salpingooophorectomy, omentectomy, terminal ileum, and right colectomy. Pathologic evaluation of the specimens revealed no evidence of malignancy. The cut section of the right adnexal mass showed an intact capsule; it was predominantly solid with cystic spaces filled with a soft yellowish material. The fallopian tube was attached to the mass. Fimbriae were not recognized. Histologically these findings corresponded with a severe acute and chronic inflammation with fibrosis, suggestive of a tubo-ovarian abscess. The gross examination of the uterus revealed a congestive mucosa and a copper IUD rigidly attached to the uterine wall up to the myometrium. Microscopically, we found acute and chronic inflammatory changes consistent with endometritis.

The colectomy specimen showed a rough fibrinoid white plaque in the serosa of the terminal ileum, cecum, and right colon. Histology revealed extensive acute inflammation with abscess formation involving the peri-colonic soft tissues and diffuse hypertrophy of the bowel's muscularis propria, with acute and chronic inflammation of both the muscularis and submucosa. There was no evidence of diverticular disease. We found sulfur granules throughout the colon and adnexal mass. Pelvic actinomycosis was diagnosed based on the acute and chronic inflammatory changes and the presence of characteristic sulfur granules (Figure 9).

The patient was evaluated every 15 days for the first 2 months after surgery; laboratory and clinical examination were normal. She was discharged from follow-up after 6 months of surveillance.

\section{Dr Odetto: Closing Summary}

Actinomycosis is an uncommon subacute or chronic progressive suppurative disease characterized by the formation of multiple

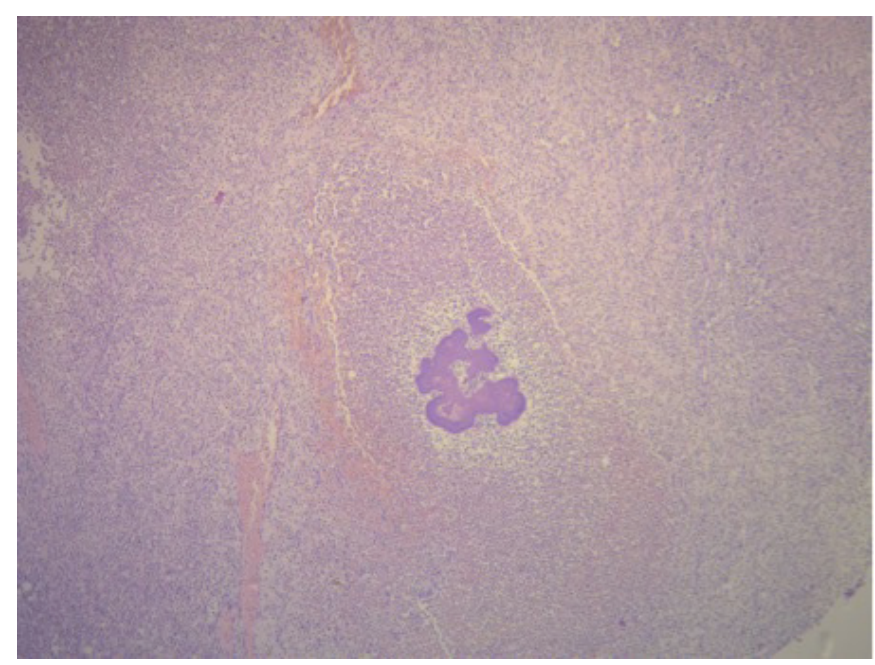

Figure 9 Microscopically, acute and chronic inflammatory changes consistent with endometritis. Sulfur granules were found throughout the colon and adnexal mass.

abscesses, abundant granulation tissue, and dense fibrous tissue, caused by anaerobic or microaerophilic bacteria. ${ }^{1}$

These two cases highlight that in the setting of prior IUD placement, pelvic actinomyces, although extremely low in frequency, may present mimicking findings consistent with either cervical or ovarian cancer.

The first case was a therapeutic challenge given the gross findings on cervical examination. On inspection, there was convincing evidence of the possibility of a primary cervical cancer. However, given the patient's history and clinical presentation, the suspicion for an inflammatory process secondary to the IUD was the most likely diagnosis. A literature search pertaining to actinomyces of the lower genital tract mimicking cervical cancer revealed only one case, but simulating a FIGO stage III, ${ }^{6}$ no case similar to the one described in this patient was found, resembling a cervical cancer FIGO stage I, treated only with antibiotics and achieving a complete response.

García et al ${ }^{7}$ studied the frequency of actinomyces in the genital tract of healthy women, in patients with cervical intraepithelial neoplasia, as well as in advanced cervical cancer. Detection of actinomyces through polymerase chain reaction was found in $9 \%$ of healthy women, and only 9\% had used an IUD at some point in their lives. In the group with cervical intraepithelial neoplasia a $10 \%$ detection rate was documented, with $52 \%$ of patients having a history of IUD use; and, lastly, $36 \%$ of patients with cervical cancer had actinomyces, with only $23 \%$ having had a history of IUD use.

Several studies have demonstrated that microbiota influence cancer susceptibility and progression by modulating inflammation, inducing oxidative stress, and promoting genomic instability of the host cells. As the bacterial density is high in mucous membranes, microbiota may also play a role in the development of pre-cancerous or cancerous lesions of the uterine cervix. ${ }^{8}$ Although Garcia et al ${ }^{7}$ demonstrated a higher prevalence of actinomyces in patients with cervical pathology when compared with healthy women, the role of this pathogen in pre-invasive and invasive cervical lesions has not been yet confirmed.

The second case corresponds to a tubo-ovarian localization, more frequently reported in the literature, especially in a patient 
with prolonged IUD use associated with a long history of abdominal pain, anorexia, weight loss, and pelvic tumor mass. ${ }^{9}$

In most reports, the diagnosis of pelvic actinomycosis occurs in women younger than 50 years old, ${ }^{10}$ and only $10 \%$ of the cases are diagnosed before surgery. First, clinical manifestations are nonspecific, consisting of fever, leukocytosis, weight loss, fatigue, and abdominal pain. Second, images are usually non-specific and nondiagnostic, and may be similar to findings for other local inflammatory or neoplastic processes. In the later stages of infection, there may be evidence of infiltration of surrounding tissues across tissue planes, with sinus tract formation that is characteristic of, but not specific to, actinomycosis. ${ }^{11}$ Finally, another reason for challenges in the pre-operative diagnosis is that direct isolation of the organism from a clinical specimen or from sulfur granules is necessary. However, this form of diagnosis occurs in only $50 \%$ of the cases for various reasons, including previous antibiotic treatment, overgrowth of concomitant organisms, or inadequate methodology.

In conclusion, pelvic actinomycosis is a disease that poses a diagnostic challenge because of its long and silent course with non-specific symptoms. In this case, this was compounded by the fact that clinical presentation mimicked cervical cancer and ovarian cancer, respectively. Antibiotic treatment based on $\beta$-lactam and a $\beta$-lactamase inhibitor (including clavulanate, benzylpenicillin, amoxicillin, ceftriaxone, meropenem, piperacillin-tazobactam, doxycycline, clindamycin, erythromycin, and clarithromycin) for at least 6 weeks should be the first therapeutic strategy. ${ }^{1213}$

Surgical treatment in these patients depends on the severity, extent, and location of disease, as well as the lack of response to antibiotics.

Correction notice This article has been corrected since it was first published. The funding statement has been amended to acknowledge the $\mathrm{NIH} / \mathrm{NCl}$ Cancer Center Support Grant P30 CA008748.

Funding This research was funded in part through the $\mathrm{NIH} / \mathrm{NCl}$ Cancer Center Support Grant P30 CA008748.
Competing interests None declared.

Patient consent for publication Not required.

Provenance and peer review Commissioned; internally peer reviewed.

Author note The authors believe this article will be of interest to all members of the gynecologic oncology community. They are appreciative of the thoughtful consideration given to their research for publication in the International Journal of Gynecological Cancer.

\section{REFERENCES}

1 Sehnal B, Beneš J, Kolářová Z, et al. Pelvic actinomycosis and IUD. Ceska Gynekol 2018;83:386-90.

2 Sanai FM, Bzeizi KI. Systematic review: tuberculous peritonitis-presenting features, diagnostic strategies and treatment. Aliment Pharmacol Ther 2005;22:685-700.

3 García-García A, Ramírez-Durán N, Sandoval-Trujillo H, et al. Pelvic actinomycosis. Can J Infect Dis Med Microbiol 2017;2017:1-17.

4 Naha K, Dasari S, Vivek G, et al. Primary abdominal nocardiosis masquerading as tubercular pelvic inflammatory disease in an immunocompetent individual. Case Rep Child Meml Hosp Chic 2013;2013.

5 Delgado G, Oram DH, Petrilli ES. Stage III epithelial ovarian cancer: the role of maximal surgical reduction. Gynecol Oncol 1984;18:293-8.

6 Snowman BA, Malviya VK, Brown W, et al. Actinomycosis mimicking pelvic malignancy. Int J Gynaecol Obstet 1989;30:283-6.

7 García-García A, Coronel-Martínez J, Leon DC-de, et al. Detection of Actinomyces spp. in cervical exudates from women with cervical intraepithelial neoplasia or cervical cancer. J Med Microbiol 2017;66:706-12.

8 Piyathilake CJ, Ollberding NJ, Kumar R, et al. Cervical microbiota associated with higher grade cervical intraepithelial neoplasia in women infected with high-risk human papillomaviruses. Cancer Prev Res 2016;9:357-66.

9 Lim DR, Hur H, Min BS, et al. Intrauterine contraceptive devicerelated actinomycosis infection presenting as ovarian cancer with carcinomatosis. Surg Infect 2014;15:826-8.

10 Hamid D, Baldauf JJ, Cuenin C, et al. Treatment strategy for pelvic actinomycosis: case report and review of the literature. Eur $J$ Obstet Gynecol Reprod Biol 2000;89:197-200.

11 Marret $\mathrm{H}$, Wagner N, Ouldamer L, et al. [Pelvic actinomycosis: just think of it]. Gynecol Obstet Fertil 2010;38:307-12.

12 Brook I. Actinomycosis: diagnosis and management. South Med J 2008;101:1019-23.

13 Trutnovsky G, Tamussino K, Reich O. Short-term antibiotic treatment of pelvic actinomycosis. Int J Gynaecol Obstet 2008;101:203-4. 


\section{Correction: Infection versus cancer: management of actinomyces mimicking cervical cancer or ovarian cancer}

Odetto D, Perrotta M, Saadi JM, et al. Infection versus cancer: management of actinomyces mimicking cervical cancer or ovarian cancer. Int J Gynecol Cancer 2020;30:1638-43.

Since the publication of this article, the following funding statement has been added: 'This research was funded in part through the NIH/NCI Cancer Center Support Grant P30 CA008748'.

(C) Author(s) (or their employer(s)) 2021. No commercial re-use. See rights and permissions. Published by BMJ. Int J Gynecol Cancer 2021;31:1400. doi:10.1136/ijgc-2020-001800corr1

A) Check for updates 